\title{
FATOR DE RISCO - ENFOQUE NA DISCIPLINA ENFERMAGEM EM CENTRO CIRÚRGICO
}

\author{
Estela Regina Ferraz Bianchi1, Brigitta E. P. Castellanos1, Maria Alice Fortes Gatto2, Arlete \\ Silva2.
}

BIANCHI, E. R. F. et alii. Fator de risco; enfoque na disciplina enfermagem em centro cirúrgico. Rev. Bras. Enf., Brasília, 39(2/3): 26-32, abr./set., 1986.

\begin{abstract}
RESUMO. Foi abordado o tema da assistência de enfermagem em centro cirúrgico dentro de uma visão do "enfoque de risco" para o paciente cirúrgico, considerando os vários riscos que podem acarretar agravos à sua saúde. Os fatores de risco foram abordados em relação ao ambiente físico, material e equipamento, pessoal e ao próprio paciente. Estes aspectos foram relacionados à área do centro cirúrgico, de recuperação anestésica e ao centro de material. O trabalho foi desenvolvido com dois grupos de alunos da Escola de Enfermagem da Universidade de São Paulo, cursando o 50 semestre de graduação, visando auxiliá-los como futuros profissionais a desenvolver uma melhor assistência de enfermagem ao paciente cirúrgico.
\end{abstract}

\begin{abstract}
The nursing surgical center discipline with an approach in the risk factor to a surgical patient considering the several risks that can cause health disorders are showed in this work. The factors risk were studied regard to the physical enviroment, material and equipment, personal and to the patient self. These aspects were affinitied with the surgical center, anesthetic recovery room and to the material center. The work was developed with two groups of students of the nursing school of São Paulo University, these students were in the fifth semester of nursing graduat course. The main objective of this work was help to the future professional to develop the best nursing care to the surgical patients.
\end{abstract}

\section{INTRODUÇÃO}

$\mathrm{O}$ ensino da assistência de enfermagem em centro cirúrgico tem sido desenvolvido como uma disciplina isolada, no 3 ? ano do curso de graduação em enfermagem, da Escola de Enfermagem da Universidade de São Paulo (EEUSP) - São Paulo. Isto traz vantagens, pois possibilita uma dedicação maior a o planejamento da assistência de enfermagem prestada ao paciente, no período peri-operatório, mas também traz desvantagens, como o não acompanhamento de toda a experiência hospitalar do paciente: desde o período pré-operatório até a recuperação pós-operatória e alta.

Tentando despertar no aluno o interesse por esta área de atuação do enfermeiro e facilitar a correlação entre a teoria e a prática da assistência de enfermagem, procuramos abordar o tema sob a forma de "enfoque de risco", uma vez que é de conhecimento que o paciente cirúrgico pertence a um grupo sujeito a vários riscos que podem acarretar agravos à sua saúde.

Numa linguagem comum, todos nós entendemos a palavra "risco" como a proximidade de um perigo ou a possibilidade de danos ou prejuízos. É preciso, entretanto, não confundir as palavras "riscos" e "danos". Consideramos "risco" como uma situação em que existe uma probabilidade de ocorrer agravos e "dano" como um resultado não desejado, uma conseqüência dos "riscos".

SUÁREZ OJEDA4 define "fator de risco" na área de saúde como "toda característica de uma pessoa ou grupo que está associada a uma probabilidade maior de aparição de um processo patológico ou de evolução especialmente desfavorável deste processo".

Um estudo adequado dentro de uma popula-

1. Professor Assistente junto à disciplina Enfermagem em Centro Cirúrgico - Escola de Enfermagem da Universidade de São Paulo.

2. Auxiliar de Ensino junto à disciplina Enfermagem em Centro Cirúrgico - Escola de Enfermagem da Universidade de São Paulo. 
ção sobre os fatores de risco a que está sujeita e sobre as conseqüencias destes riscos traz elementos importantes para o planejamento e execução da assistência de enfermagem, possibilitando, também, a detecção dos grupos de maior risco dentro desta população, dando, assim, um sentido de prevenção às ações de enfermagem.

A partir do conceito de risco em saúde apresentado por CARVALHO ${ }^{1}$, consideramos risco na área da assistência de enfermagem em centro cirúrgico como a probabilidade de um indivíduo de apresentar um agravo em seu processo saúde-doença que já está comprometido pela afecção que o levou à necessidade de um tratamento cirúrgico e que será mais agravado pelo procedimento anestésico-cirúrgico.

Segundo ainda CARVALHO ${ }^{1}$, alguns fatores de risco são intrínsecos às pessoas e outros são gerados pelo meio e podem "abrir perspectivas para a prevenção e o controle dos danos que podem incidir na clientela das instituições de saúde, sob a assistência do pessoal de enfermagem". Chegamos à conclusão de que o enfoque de fator de risco deveria ser abordado em relação a: ambiente físico; material e equipamento; pessoal e os relacionados ao paciente. Esse fatores de risco foram por nós estudados e discutidos na área de centro cirúrgico e na de recuperação pósanestésica. $\mathrm{Na}$ área de centro de material foram estudados ambiente, material e equipamento e foram acrescentados os referentes ao funcionário que atua nessa área hospitalar.

Após o preparo destas aulas, consideramos que os fatores de risco relacionados ao ambiente físico e ao material e equipamento ficariam para serem desenvolvidos por cada grupo de estágio (4 a 8 alunos), durante o período em que permanecessem nessa área, e apresentados à classe sob forma de seminários. Foi bastante gratificante verificar, em cada uma destas apresentações, como os alunos captaram a essência dessa abordagem de risco, demonstrando terem conseguido uma visão concreta dos possíveis riscos de cada um dos ambientes em que estavam desenvolvendo seu trabalho de campo.

Os demais fatores de risco, isto é, os relacionados ao pessoal e à assistência prestada ao paciente, foram apresentados sob forma de preleção com discussão para o grupo I; para o grupo II, alguns itens como os relacionados aos componentes do trauma cirúrgico, foram estudados em grupo pelos alunos $\mathrm{e}$ apresentados à classe sob forma de discussão.

Para cada fator de risco foram definidos:

a) indicador: situação que leva a um fator de risco;

b) ocorrência: o que é percebido como conseqüência do indicador ou da causa no paciente, ambiente, pessoal, material ou equipamento;

c) causa: o que ocasionou o indicador e/ou a ocorrência.

Esses parâmetros (indicador, ocorrência e causa) que foram colocados na análise de cada fator de risco necessitam de mais reflexões pois dúvidas ainda permaneceram, levando a certa dificuldade do aluno em compreender claramente esta abordagem do enfoque de fator de risco. Por ser usada como método de ensino há, também, necessidade da atuação do enfermeiro em cada situação de risco e sua análise minuciosa. Esse aspecto não foi sistematicamente abordado com esses dois grupos de alunos havendo necessidade de maior aprofundamento do tema.

A título de exemplo, podemos citar o seguinte fator de risco:

1. fator de risco - paciente

2. indicador - alteração da percepção sensorial e função neurológica

3. ocorrências:

- diminuição ou ausência da audição e visão

- diminuição ou aumento da dor

- alteração do nível de consciência

- alteração endócrino-metabólica

- insuficiência respiratória (principalmentete a respiração profunda na dor)

- alteração motora-sensorial

- dificuldade de adaptação ao ambiente de centro cirúrgico, sala de recuperação pósanestésica

- insegurança física e emocional

4. causas (da ocorrência):

- comprometimento neurológico

- problemas auditivos, visuais, sensoriais

- falha na individualização de cuidados

- ação dos anestésicos usados

- disfunção neurológica com conseqüente alteração endócrino-metabólica

- alteração dos componentes psicológicos e fisiológicos da dor.

(do indicador)

- afecção dos órgãos sensoriais e sistema nervoso.

Sabemos que esse foque de "fator de risco" tem tido maior aplicação na área de saúde da comunidade, prevenindo agravos simples à saúde, como aborda NOGUEIRA 3 : "o conceito de risco é, então, e m princípio, u m dos principais meios para o estabelecimento de um sistema de assistência primária de saúde". Outra abordagem encontrada do enfoque de risco é em relação ao grau de perdas financeiras para o cliente e/ou instituição, como aponta DAVIS 2 . $\mathrm{O}$ método empregado para diminuir esse risco financeiro é o levantamento do fator essencial e a sua prevenção. Esse mesmo autor relata um estudo feito com 20.864 pacientes onde foram identificados 970 eventos como "riscos potenciais" sendo que o centro cirúrgico foi apontado em $71,8 \%$ dos casos. São citados como exemplos destes riscos em centro cirúrgico: objetos estranhos deixados no paciente durante a cirurgia; erros de medicação, equipamento sem a devida manutenção; perfuração de órgãos; perda de biópsia, cultura, peça; perda de documentos e regis tros; queimaduras; infecção pós-operatória veiculada ao periodo transoperatório; reação indesejada a drogas; problemas com normas, procedimentos e regulamento.

Correlacionando esses problemas citados com a nossa realidade de assistência de enfermagem em centro cirúrgico, cremos ser importante a abordagem e discussão desses fatores de risco com os alunos do curso de graduação em enfermagem. 
Cabe-nos ressaltar que os campos de estágio usados pela nossa disciplina são: pequena cirurgia e centro cirúrgico, centro de material, e sala de recuperação pós-anestésica.

Numa tentativa de avaliação do processo ensino-aprendizagem desenvolvido com o auxílio deste enfoque de risco à assistência de enfermagem no período peri-operatório, a dois grupos de alunos do curso de graduação em enfermagem, realizamos um levantamento das opiniões e sugestões quanto a este enfoque e quanto à utilização em outras disciplinas.

\section{OBJETIVOS}

Verificar, junto aos alunos do 50 semestre do curso de graduação em enfermagem, sua opinião quanto à abordagem de fator de risco na disciplina Enfermagem em Centro Cirúrgico.

Verificar, junto aos alunos do 50 semestre do curso de graduação em enfermagem, a utilização dessa abordagem em outra disciplina cursada.

Levantar, junto aos alunos do 50 o semestre do curso de graduação em enfermagem, sugestões para melhorar o ensino da abordagem do enfoque de risco na disciplina Enfermagem em Centro Cirúrgico.

\section{MATERIAL E MÉTODO}

\section{População}

A população deste estudo foi constituída por 48 alunos do 50 semestre do curso de graduação em enfermagem, da EEUSP, do corrente ano, sendo que 27 alunos cursaram a disciplina no bimestre de marçoabril e 21 alunos e fizeram em maio-junho.

Coleta de dados

$\mathrm{O}$ instrumento utilizado para a coleta de dados foi o questionário.

Para os alunos do grupo I (aqueles que cursaram a disciplina no período de março-abril), o questionário constou de peiguntas fechadas quanto à adequação do enfoque de fator de risco para a aprendizagem e quanto à adequação deste enfoque a outra disciplina; uma pergunta aberta para verificar as sugestões quanto a esta abordagem.

Para os alunos do grupo II (aqueles que cursaram a disciplina no período de maio-junho), o questionário foi o mesmo aplicado ao grupo I, com a eliminação das perguntas fechadas relacionadas à adequação do enfoque de risco à outra disciplina, porque Enfermagem em Centro Cirúrgico foi a última disciplina cursada no semestre.

\section{Análise e apresentação dos resultados}

A análise dos dados foi feita qualitativamente e utilizando-se índices percentuais.

Os dados serão apresentados levando-se em consideração:

a) características da amostra;

b) opinião dos alunos quanto à adequação do do enfoque de risco à aprendizagem na disciplina Enfermagem em Centro Cirúrgico;

c) opinião dos alunos quanto à adequação do enfoque de risco à aprendizagem de outra disciplina cursada; d) sugestões dos alunos quanto à abordagem de enfoque de risco.

\section{RESULTADOS E DISCUSSÃO DOS DADOS}

\section{Caracteristicas da amostra}

A amostra levantada correspondeu a 21 $(100,0 \%)$ dos alunos do grupo II e a $24(88,8 \%)$ dos alunos do grupo I, perfazendo 45 alunos. Todos os alunos que estavam presentes à sala de aula, no dia da aplicação do questionário, responderam às perguntas formuladas.

Opinião dos alunos quanto à adequação do enfoque de risco à aprendizagem na disciplina Enfermagem em Centro Cinirgico

Acreditando que a abordagem de fator de risco é importante para a aprendizagem da assistência de enfermagem nessa área, pelos alunos do curso de graduação em enfermagem, levantamos os pontos que consideramos essenciais e solicitamos aos alunos que avaliassem cada item, obecendo os seguintes critérios:

meu aprendizado não foi facilitado por esta metodologia;

meu aprendizado não dependeu desta metodologia;

meu aprendizado foi facilitado por esta metologia.

Pela análise das respostas, obtivemos os seguintes resultados que são apresentados sob forma de tabelas.

Pelos dados da Tabela 1, podemos verificar que os alunos do grupo I opinaram positivamente sobre a aprendizagem baseada em fator de risco: 23 alunos $(95,8 \%)$ desenvolveram a percepção global do centro de material; 22 alunos $(91,6 \%)$ perceberam o desenvolvimento da análise crítica; 19 alunos $(79,1 \%)$ encontraram facilidade em desenvolver a observação sistematizada, em aplicar a teoria proposta ao trabalho de campo e, também, em desenvolver a percepção global do centro cirúrgico.

Podemos, portanto, concluir que os alunos do grupo I classificaram como facilitadora a abordagem do fator de risco em relação aos tópicos levantados no ensino de Enfermagem em Centro Cirúrgico. Isto vem de encontro à nossa crença de que essa abordagem proporciona condições aos alunos para terem visão global do que acontece nessa área, procurando desenvolver a análise crítica e não ficando somente se detendo na prestação mecânica e automática da assistência de enfermagem ao paciente, mas também, inter-relacionando os fatores inerentes ao ambiente, material, equipamento, e pessoal.

Essa nossa preocupação tem bases concretas, pois, na realidade da prática profissional, verificamos que o enfermeiro de centro cirúrgico geralmente está preocupado com o material e equipamento, sendo chamado para atuar como "bombeiro", ficando a prestação da assistência de enfermagem delegada ao circulante de sala. Acreditamos que devemos enfatizar a área expressiva de atuação do enfermeiro, isto é, a assistência de enfermagem ao pacien- 
TABELA 1 - Distribuição da opinião dos alunos do grupo I, em relação à aprendizagem dessa metologia na disciplina Enfermagem em Centro Cirúrgico. São Paulo, 1985.

\begin{tabular}{|c|c|c|c|c|c|c|c|c|}
\hline \multirow{2}{*}{$\begin{array}{l}\text { AVALIAÇÃO } \\
\text { APRENDIZADO }\end{array}$} & \multicolumn{2}{|c|}{1} & \multicolumn{2}{|c|}{2} & \multicolumn{2}{|c|}{3} & \multicolumn{2}{|c|}{ TOTAL } \\
\hline & Nọ & $\%$ & Nọ & $\%$ & Nọ & $\%$ & Nọ & $\%$ \\
\hline $\begin{array}{l}\text { desenvolvimento de } \\
\text { observação sistematizada }\end{array}$ & 4 & 16,7 & 1 & 4,2 & 19 & 79,1 & 24 & 100,0 \\
\hline $\begin{array}{l}\text { desenvolvimento de } \\
\text { análise crítica }\end{array}$ & 1 & 4,2 & 1 & 4,2 & 22 & 91,6 & 24 & 100,0 \\
\hline $\begin{array}{l}\text { aplicação da teoria no } \\
\text { trabalho de campo }\end{array}$ & - & - & 5 & 20,8 & 19 & 79,2 & 24 & 100,0 \\
\hline $\begin{array}{l}\text { desenvolvimento da } \\
\text { percepção global de } \\
\text { centro cirúrgico }\end{array}$ & 1 & 4,2 & 4 & 16,7 & 19 & 79,1 & 24 & 100,0 \\
\hline $\begin{array}{l}\text { desenvolvimento da } \\
\text { percepção global de } \\
\text { centro de material }\end{array}$ & 1 & 4,2 & - & - & 23 & $\dot{95,8}$ & 24 & 100,0 \\
\hline $\begin{array}{l}\text { desenvolvimento da } \\
\text { percepção global da } \\
\text { S.R.P. Anestésica* }\end{array}$ & 2 & 8,4 & 5 & 20,8 & 17 & 70,8 & 24 & 100,0 \\
\hline $\begin{array}{l}\text { desnvolvimento da } \\
\text { percepção global da } \\
\text { pequena cirurgia }\end{array}$ & 1 & 4,2 & 5 & 20,8 & 18 & 75,0 & 24 & 100,0 \\
\hline
\end{tabular}

"sala de recuperação pós-anestésica.

te, mas não podemos deixar os alunos sem a visão da totalidade que engloba essa assistência correlacionando esses fatores externos, muitas vezes de máxima importância para a compreensão do que ocorre com o paciente.

Ainda analisando os dados da Tabela 1 , verificamos que alguns dos alunos do grupo I classificaram como independente da abordagem de fator de risco a aprendizagem dos tópicos: aplicação da teoria no trabalho de campo - 5 alunos $(20,8 \%)$ e no desenvolvimento da percepção global da sala de recuperação pós-anestésica e da pequena cirurgia - 5 alunos $(20,8 \%)$; relataram a não interferência dessa metodologia na aprendizagem do desenvolvimento da percepção global de centro cirúrgico -4 alunos $(16,7 \%)$.

Verificamos, também, que, para alguns alunos do grupo I, essa metodologia não facilitou a aprendizagem e desenvolvimento da observação sistematizada - 4 alunos $(16,7 \%)$, e no desenvolvimento da percepção global do centro cirúrgico -2 alunos $(8,3 \%)$.

Refletindo sobre estes dados, podemos concluir que essa metodologia, para os alunos do grupo I, facilitou a aprendizagem e auxiliou na percepção da complexidade das unidades de centro cirúrgico, centro de material e recuperação pós-anestésica.
Analisando os dados da tabela 2, podemos verificar que os alunos do grupo II avaliaram esta metodologia como facilitadora para a aprendizagem dos itens: desenvolvimento da percepção global de sala de recuperação pós-anestésica - 20 alunos $(95,2 \%)$; da percepção global de centro de material - 19 alunos $(90,5 \%)$ e do centro cirúrgico - 18 alunos $(85,7 \%)$.

Podemos constatar que esta metodologia de enfoque de risco aplicada à assistência de enfermagem também foi apreciada pelo grupo II, como um meio para facilitar o ensino e aprendizagem dos principais aspectos das unidades de centro cirúrgico, centro de material e recuperação pós-anestésica.

Os alunos do grupo II avaliaram como independente a sua aprendizagem dos itens: desenvolvimento da análise crítica - 9 alunos $(42,9 \%)$; aplicação da teoria no trabalho de campo -6 alunos $(28,6 \%)$, e no desenvolvimento de observação sistematizada e de percepção global da pequena cirurgia -3 alunos $(14,3 \%)$.

A aprendizagem dos alunos do grupo II, como podemos também verificar na tabela 2 , não foi facilitada, quanto ao desenvolvimento da observação sistematizada e da percepção global de centro de material, somente por 2 alunos $(9,5 \%)$.

Para os dois grupos pesquisados, podemos constatar que a abordagem do enfoque de risco foi consi- 
TABELA 2 - Distribuição da opinião dos alunos do grupo II, em relação à aprendizagem dessa metodologia na disciplina Enfermagem em Centro Cirúrgico. São Paulo, 1985.

\begin{tabular}{|c|c|c|c|c|c|c|c|c|}
\hline \multirow{2}{*}{$\begin{array}{l}\text { APRENDIZADO } \\
\text { OBTIDO }\end{array}$} & \multicolumn{2}{|c|}{1} & \multicolumn{2}{|c|}{2} & \multicolumn{2}{|c|}{3} & \multicolumn{2}{|c|}{ TOTAL } \\
\hline & Nọ & $\%$ & Nọ & $\%$ & № & $\%$ & Nọ & $\%$ \\
\hline $\begin{array}{l}\text { desenvolvimento de } \\
\text { observação sistematizada }\end{array}$ & 2 & 9,5 & 3 & 14,3 & 16 & 76,2 & 21 & 100,0 \\
\hline $\begin{array}{l}\text { desenvolvimento de } \\
\text { análise crítica }\end{array}$ & 1 & 4,8 & 9 & 42,9 & 11 & 52,3 & 21 & 100,0 \\
\hline $\begin{array}{l}\text { aplicação da teoria } \\
\text { no trabalho de campo }\end{array}$ & - & - & 6 & 28,6 & 15 & 71,4 & 21 & 100,0 \\
\hline $\begin{array}{l}\text { desenvolvimento da } \\
\text { percepção global de } \\
\text { centro cirúrgico }\end{array}$ & 1 & 4,8 & 2 & 9,5 & 18 & 85,7 & 21 & 100,0 \\
\hline $\begin{array}{l}\text { desenvolvimento da } \\
\text { percepção global de } \\
\text { centro de material }\end{array}$ & 2 & 9,5 & - & - & 19 & 90,5 & 21 & 100,0 \\
\hline $\begin{array}{l}\text { desenvolvimento da } \\
\text { perceção global da } \\
\text { s.r.p. anestésica* }\end{array}$ & - & - & 1 & 4,8 & 20 & 95,2 & 21 & 100,0 \\
\hline $\begin{array}{l}\text { desenvolvimento da } \\
\text { percepção global da } \\
\text { pequena cirurgia }\end{array}$ & 1 & 4,8 & 3 & 14,3 & 17 & 81,0 & 21 & 100,0 \\
\hline
\end{tabular}

*sala de recuperação pós-anestésica.

derada facilitadora da aprendizagem, principalmente quanto ao desenvolvimento da percepção global do centro de material.

Consideramos que a aplicação da teoria no trabalho de campo, apontada por ambos os grupos como um tópico independente da metodologia aplicada, mantém íntima relação com as oportunidades que surgem para os alunos efetuarem um trabalho de intervenção concreta no campo de estágio. Geralmente, o aluno de curso de graduação precisa ter um tempo para se adaptar a esta unidade fechada e com rigor de técnica asséptica e, com o rodízio desenvolvido pela disciplina nos diversos campos, o estudante fica, muitas vezes, com poucas oportunidades de aplicar alguns conhecimentos obtidos.

Aceitamos como desafio trabalhar esses tópicos levantados como independentes e como não facilitados pela metodologia empregada, o que já estamos procurando melhorar para o grupo do 20 semestre.

Opinião dos alunos quanto à adequação do enfoque de risco na aprendizagem de outra disciplina cursada.

Os alunos do grupo I cursaram a disciplina Enfermagem em Centro Cirúrgico no 19 bimestre de 1985 , cursando no 20 bimestre outra disciplina, razão pela qual pudemos verificar qual a opinião desse grupo quanto à utilização da metodologia do enfoque de risco na assistência de enfermagem aprendida na disciplina Enfermagem em Centro Cirúrgico, durante o desenvolvimento dessa disciplina subseqüente.

Neste sentido, propusemos algumas situações que acreditamos serem importanes na aplicação desse enfoque, as quais são apresentadas a seguir.

Pelos dados da Tabela 3, verificamos que os alunos do grupo I aplicaram os conhecimentos da metodologia de enfoque de risco principalmente na observação sistematizada do ambiente - 22 alunos $(91,7 \%)$; na observação sistematizada do material e equipamento - 22 alunos $(91,7 \%)$; na observação sistematizada dos recursos humanos - 19 alunos $(79,2 \%)$; na análise crítica da assistência de enfermagem prestada - 18 alunos $(75,0 \%)$, e na observação sistematizada da organização do serviço -17 alunos $(70,8 \%)$.

Com base nesses dados, podemos concluir que uma aplicação para a metodologia de enfoque de risco na aprendizagem dos alunos é, como esperávamos, na observação global e crítica do ambiente, do material e equipamento, do pessoal, da assistência de enfermagem prestada e da organização do serviço.

Os alunos não perceberam muito a aplicação deste enfoque na observação sistematizada do cliente - 15 alunos (62,5\%); no planejamento de assistên- 
TABELA 3 - Distribuição da opinião dos alunos do grupo I, em relação à posterior aplicação da metodologia de enfoque de risco na assistência de enfermagem. São Paulo, 1985.

\begin{tabular}{l|rr|rr|rr}
\hline APLICAÇÃO POSTERIOR DA ABORDAGEM & \multicolumn{2}{|c|}{ SIM } & \multicolumn{2}{c|}{ NÃO } & \multicolumn{3}{c}{ TOTAL } \\
TÓPICOS ABORDADOS & No & $\%$ & No & $\%$ & No & $\%$ \\
\hline observação sistematizada do ambiente & 22 & 91,7 & 2 & 8,3 & 24 & 100,0 \\
$\begin{array}{l}\text { observação sistematizada do material e } \\
\text { equipamento }\end{array}$ & 22 & 91,7 & 2 & 8,3 & 24 & 100,0 \\
observação sistematizada dos recursos humanos & 19 & 79,2 & 5 & 20,8 & 24 & 100,0 \\
$\begin{array}{l}\text { observação sistematizada da organização } \\
\text { do serviço }\end{array}$ & 17 & 70,8 & 7 & 29,2 & 24 & 100,0 \\
observação sistematizada do cliente & 9 & 37,5 & 15 & 62,5 & 24 & 100,0 \\
$\begin{array}{l}\text { análise crítica da assistência de enfermagem } \\
\text { prestada }\end{array}$ & 18 & 75,0 & 6 & 25,0 & 24 & 100,0 \\
planejamento da assistência de enfermagem & 12 & 50,0 & 12 & 50,0 & 24 & 100,0 \\
intervenção de enfermagem & 13 & 54,2 & 11 & 45,8 & 24 & 100,0 \\
\hline
\end{tabular}

cia de enfermagem - 12 alunos $(50,0 \%)$; e na intervenção de enfermagem -11 alunos $(45,8 \%)$.

\section{- Sugestões dos alunos quanto à abordagem de enfoque de risco.}

Essa questão foi formulada com a finalidade de levantarmos as sugestões dos alunos. Verificamos que, dos $45(100,0 \%)$ alunos que participaram do estudo, $24(53,3 \%)$ não a responderam. Podemos considerar que os alunos acharam importante e até gostaram de como foi feita a abordagem, ou então, não gostaram mas também não tinham o que sugerir, ou não quiseram dar sugestões, ou a questão estava mal formulada.

Dos 21 alunos $(46,7 \%)$ que manifestaram sugestões, 10 foram quanto à abordagem do enfoque de risco e 11 foram em relação à disciplina como um todo. Alguns alunos deram tanto sugestões em relação à abordagem, como em relação à disciplina. Isto vem reforçar o nosso pensamento de que esta questão ficou muito ampla, dando margem a dúvidas. Somente consideramos as sugestões referentes à metodologia de enfoque de risco. Elas foram agrupadas e colocadas no Quadro 1, a seguir.

QUADRO 1 - Distribuição das sugestões manifestadas por 10 alunos quanto à metodologia de enfoque de risco. São Paulo, 1985 .

\begin{tabular}{cr}
\hline SUGESTÕES & Nọ \\
\hline
\end{tabular}

melhoria da abordagem, em classe, do enfoque de risco

focalizar também a criança e o recém-nascido exigir maior conhecimento do aluno

aplicação em trabalho feito no campo de estágio

não utilizar o indicador na abordagem em classe

estudo dos fatores de risco no decorrer do estágio

apresentação de todos os fatores de um campo de estágio para evitar repetição

aula teórica e, em paralelo, a discussão de fator de risco

maiores possibilidades para discussão em sala de aula

TOTAL

\section{CONCLUSÕES}

Pela análise dos dados, podemos concluir que os alunos alvo deste levantamento sobre a abordagem do enfoque de risco aplicada na disciplina Enfermagem em Centro Cirúrgico avaliaram este método como facilitador, nos seguintes tópicos:

Grupo I: percepção global do trabalho desenvolvido nas áreas de centro de material $(95,8 \%)$, centro cirúrgico $(79,2 \%)$, pequena cirurgia $(75,0 \%)$ e recuperação pós-anestésica $(70,8 \%)$; desenvolvimento da análise crítica $(91,2 \%)$; observação sistematizada e a aplicação da teoria proposta no trabalho de campo $(79,2 \%)$. 
Grupo II: desenvolvimento da percepção global de recuperação pós-anestésica $(95,2 \%)$, centro de material $(90,5 \%)$, centro cirúrgico $(85,7 \%)$ e pequena cirurgia $(81,0 \%)$; desenvolvimento de observação sistematizada $(76,2 \%)$; aplicação da teoria no trabalho de campo $(71,4 \%)$ e desenvolvimento da análise crítica $(52,4 \%)$.

A opinião dos alunos em relação à aplicação desta metodologia na aprendizagem em outra disciplina cursada foi favorável quanto a: observação sistematizada do ambiente, material e equipamento da assistência de enfermagem prestada $(75,0 \%)$. Alguns alunos não aplicaram esta metodologia na observação sistematizada do cliente $(62,5 \%)$.

Dentre os alunos, 10 manifestaram sugestões para a melhoria do método de enfoque de risco, relacionadas a discussões e forma de apresentação em sala de aula e a maior aplicação nos campos de estágio.

\section{CONSIDERAÇÕES FINAIS}

Nós, como docentes, acreditamos que temos papel primordial no desenvolvimento das potencialidades dos alunos, auxiliando-os, como futuros profissionais, a cada vez mais melhorarem a assistência de enfermagem prestada. Portanto, devemos procurar abordagens de conteúdo programático que façam com que o aluno desenvolva observação global e sistematizada da área onde está atuando tendo como centro a prestação de cuidados de enfermagem ao paciente.

Sabemos que a unidade de centro cirúrgico, englobando aqui o centro de material e recuperação pós-anestésica, é considerada por alguns alunos como amedrontadora e, muitas vezes, até desumana, ficando assim, mais uma vez, reforçada a importância dessa metodologia para incentivar e facilitar a aprendizagem do aluno e sua atuação num futuro próximo.

Acreditamos que este trabalho foi o início da reflexão, análise crítica e implementação da metologia de enfoque de risco, para a atuação do estudante nos campos de estágio nesta perspectiva de abordagem da assistência de enfermagem, num trabalho em equipe com o docente e demais enfermeiros, com vistas ao alcance de uma competência profissional cada vez maior, medida pelos benefícios proporcionados aos seus pacientes.

BIANCHI, E. R. F. et alii. Risk factor; an approach in the nursing surgical center discipline. Rev. Bras. Enf., Brasília, 39(2/3): 26-32, Apr./Sept ., 1986.

\section{REFERÉNCIAS BIBLIOGRÁFICAS}

1. CARVALHO, J. F. Modelo operacional de enfermagem com enfoque preventivo. Brasilia, $\mathrm{ABEn}, 56 \mathrm{p}$. mimeografado.

2. DAVIS, D. Risk management: another OR puzzle to solve? AORN J., Denver, 38 (5) : 767-71, Nov. 1983.

3. NOGUEIRA, M. J. de C. Enfoque de risco na assistência de enfermagem comunitária. Rev. Esc. Enf. USP., São Paulo, 18 (3): 271-85, dez. 1984.

4. SUÁREZ OJEDA, E. N. El enfoque de riesgo en la atención perinatal y maternoinfantil. Bol. Of. Sanit. Panam., Washington, 92 (6): 482-93, jun. 1982. 